\title{
Development of Health Mix from Silverbellies and their Nutritional Characteristics
}

\author{
K. Rathnakumar ${ }^{1 *}$ and N. Pancharaja ${ }^{2}$ \\ ${ }^{1}$ Department of Fish Process Engineering, College of Fisheries Engineering, Tamil Nadu \\ Fisheries University, Nagapattinam-611002, Tamil Nadu, India \\ ${ }^{2}$ Department of Fisheries, Tenampet, DMS complex, Chennai, Tamil Nadu, India \\ *Corresponding author
}

\section{A B S T R A C T}

Keywords

Silverbellies, Acid

hydrolysis, Fish powder -

cereal mixture,

Nutritional characteristics

Article Info

Accepted:

26 February 2018

Available Online:

10 March 2018
Minced Silverbellies fish meat (Leiognathus sp.) was subjected to steam cooking and acid hydrolysis and dried to a moisture content of 6-7\%. Fish meat powder obtained from both methods were separately incorporated at different percentages $(10 \%, 15 \%, 20 \%$ and $25 \%)$ into a cereal mixture containing malted and roasted wheat and ragi powder. Prepared fish powder - cereal mixture were stored at ambient temperature and their storage characteristics like biochemical composition, nutritional, functional, microbiological parameters and organoleptical attributes were analyzed upto 90 days. Drinks prepared by using hydrolysed fish powder were organoleptically more acceptable, $(\mathrm{P}<0.01)$

\section{Introduction}

Fisheries make an important contribution to the world population by providing animal protein which is of great significance. Among the various types of fishing, shrimp trawling is the bigger contributor with the highest ratio of by-catch to shrimp catch about 10:1 in tropical waters (Sahoo, 2007). The by-catch fishes are also good in nutritional point of view (Anon, 2001). Low value by-catch contains small bony fishes like silverbellies, anchovies, lizard fish etc. Among these silverbellies contributed to $10 \%$ of the total marine landings during 2005-2006 (CMFRI, 2006). There is an increasing demand for fish and fish products around the world due to its health benefits roles (Feldhusen, 2000). Seafood is one of the highest quality protein supplements available at low cost. To meet the increasing demand for fish, development of new products from underutilized fish species are taken up. To develop a new product, it is essential to know its storage behaviour. In the present investigation cereal mixture mixed with fish powder was prepared and its properties were studied.

\section{Materials and Methods}

Silverbellies (Leiognathus sp) caught off Thoothukudi coast brought under iced 
condition was used for present study. Minced meat obtained using deboner $(\mathrm{M} / \mathrm{s}$. Badder, Germany) was washed in chilled water and divided into two parts. One part was steam cooked for 10 minutes and another part was hydrolysed by using $1 \mathrm{~N} \mathrm{HCl}$ (Setty et al., 1977) for 12 hours and neutralized with $1 \mathrm{~N}$ sodium hydroxide and the meat was washed alternatively with hot and cold water for 4 to 5 times and dried at $60^{\circ} \mathrm{C}$ using electrical oven until moisture content reaches 6-7\%. Hydrolysed meat was neutralized to $\mathrm{pH} 7$ using $1 \mathrm{~N} \mathrm{NaOH}$ and thoroughly water washed to remove the traces of alkali. Water washed cereals (Wheat and ragi) were soaked in water for 6 hours. After draining cereals were covered with cloth for 36 hours with occasional water spray. Sprouted cereals were dried, roasted, dehusked and powdered. Both cooked and hydrolysed fish powder was mixed with cereal mix containing malted and roasted wheat and ragi powder (equal volume) at different percentage $(10 \%, 15 \%, 20 \%$ and $25 \%$ ), and packed in aluminium foil laminated pouches. Storage behaviour of the product was studied up to 90 days.

Moisture, protein, fat, ash, peroxide value, $(\mathrm{PV})$, calcium and pepsin digestibility were determined as per standard methods of AOAC (1995). Phosphorus was estimated by the method of Fiske and Subbarow (1925). $\mathrm{Ca}^{2+}$ ATPase activity (Noguchi and Matsumoto, 1970), Solubility (Jeyakumari et al., 2006), pH of the sample with digital $\mathrm{pH}$ meter (335, systronics, India) and viscosity with digital viscometer (Brookfield, U.S.A) were determined. Carbohydrate and available lysine were determined by the method of Sadasivam and Manickam (1992). Free fatty acid (FFA) was estimated by the method of Olley and Lovern (1960). Total volatile base nitrogen (TVB-N) were estimated by the procedure of Beatty and Gibbons (1937) using Conway's micro diffusion technique. Fat absorption capacity (FAC) was estimated by the method of Lin et al., (1974). Water absorption capacity (WAC) was determined by the method of Solsulski (1962) and microbiological parameters such as TPC, E. coli, Staphylococcus, Salmonella and Vibrio cholera were determined by the method of APHA (1976). Organoleptical quality of the fish powder - cereal mixtures was evaluated by preparing a drink. One spoon of mixture $(10-15 \mathrm{~g})$ was mixed in hot water or milk of $200 \mathrm{ml}$ with required amount of sugar. This drink was subjected to sensory qualities test such as appearance, colour, odour and overall acceptability by a trained panelist. The panelists were asked to determine the attribute of quality on the basis of 5-point scale. Correlation (Snedecor and Cochran, 1967) was done with respect to storage period and other parameters.

\section{Results and Discussion}

In the present study the average length and weight of silverbellies used were $9.6 \pm 0.32 \mathrm{~cm}$ and $14.5 \pm 0.56 \mathrm{~g}$ respectively. The yield of minced meat from whole fish was $35 \%$ and Revankar et al., (1981) have reported 34 $50 \%$ of yield from pink perch. The result in the present study is in agreement with reasonable limit. Generally the yield of whole fish is directly related to the size of fish and season. The yield of fish meat powder was about $10.76 \%$ on cooking and $9.75 \%$ on hydrolysis. Physico-chemical, functional and microbiological characteristics of silverbellies mince are presented in Table 1 . In fresh meat TMA-N and PV were absent which could be due to repeated washing of meat at $4^{\circ} \mathrm{C}$. Fresh meat had $16.38 \%$ protein. Higher solubility $(90.05 \%)$, viscosity (11.35 cp) and $\mathrm{Ca}^{++}$ ATPase activity at $0.79 \mu \mathrm{g} \mathrm{pi} / \mathrm{mg}$ protein $/ \mathrm{min}$ indicated freshness and conformational status of myofibrillar proteins. Several authors have reported on the proximate composition and its nutritive value of silverbellies (Srinivasan, 1966, Venugopalan and James (1969) and 
Chattopadhyay et al., (2004). The result in the present study is in agreement with earlier report in fresh meat. Total plate count (TPC) of $1.15 \times 10^{3} \mathrm{cfu} / \mathrm{g}$, Staphylococci and E. coli were found to be $7 \mathrm{cfu} / \mathrm{g}$ and $3 \mathrm{cfu} / \mathrm{g}$ respectively were observed. Salmonella and vibrio were absent.

The physico-chemical and functional properties of fish meat powder from silverbellies prepared by both steam cooking and hydrolysis process are given in Table 2. The protein content of fish powder from cooked and hydrolyzed meat was $85.52 \%$ and $84.82 \%$ respectively. Available lysine and pepsin digestibility of the above powders were $9.94 \%$ and $9.62 \%$ respectively. Calcium and phosphorus contents of cooked meat powder were found to be $344.65(\mathrm{mg} / 100 \mathrm{~g})$ and $390.81(\mathrm{mg} / 100 \mathrm{~g})$ respectively, whereas in hydrolysed meat powder, their contents were $380.11(\mathrm{mg} / 100 \mathrm{~g})$ and $410.82(\mathrm{mg} / 100 \mathrm{~g})$ respectively. The values of TVB-N, FFA and PV were found to be 1.35 ( $\mathrm{mg} \%), 0.01 \%$ of oleic acid and 0.14 milli equivalent $\mathrm{O}_{2} / \mathrm{kg}$ of fat respectively in cooked meat powder. In hydrolysed meat powder their values were $1.32 \mathrm{mg} \%, 0.009 \%$ of oleic acid and 0.11 milli equivalent $\mathrm{O}_{2} / \mathrm{kg}$ of fat respectively. TVB-N, FFA and PV values of hydrolysed meat powder did not vary significantly with cooked meat powder. Similar result reported by Srinivasan (1966) that fish meat of silverbellies has good protein content and high pepsin digestibility. Edible fish powder prepared by Chattopadhyay et al., (2004) from silverbellies had very high content of calcium and phosphorus.

During storage period proximate composition did not vary significantly in the products developed by fortifying cereals with varying percentage of fish powder (cooked and hydrolysed). However protein content increased with increase in amount of fish powder, with a corresponding decrease in the carbohydrate content. A higher protein content
$(27.91 \%)$ was found in $25 \%$ cooked fish powder incorporated mixture, where as it was $(24.95 \%)$ in $25 \%$ hydrolysed meat powder incorporated mixture. Similar results observed in gelatinized product prepared from rice and wheat flour (Jeyakumari and Rathnakumar, 2006).

The results for changes in nutritional characteristics of both fish powder -cereal mixture are presented in Table 3 and 4. The value of calcium, phosphorus, available lysine and pepsin digestibility were found to be increase with increasing amount of incorporated fish powder. A higher value of available lysine $\left(7.4 \mathrm{~g} \pm 0.56 \mathrm{~g} / 16 \mathrm{gN}_{2}\right)$ and pepsin digestibility $(91.57 \pm 0.75 \%)$ were found in $25 \%$ cooked meat powder incorporated mixture, whereas, higher values of calcium and phosphorus (236.52 \pm 0.93 and $323.27 \pm 0.83 \mathrm{mg} / 100 \mathrm{~g}$ ) was recorded in $25 \%$ hydrolyzed meat powder incorporated mixture.

The values of calcium and phosphorus increased with increasing amount of fish powder incorporation. Setty et al., (1977) reported that partially hydrolysed and deodourised fish flour had 7-8 \% available lysine. During storage period the nutritive value of health mix did not vary significantly $(\mathrm{P}<0.01)$. The results for changes in functional properties of fish powder - cereal mixture are presented in Table 5 and 6 . The value of WAC and FAC were found to increase with increasing amount of added fish powder. However, in the present study the WAC of meat powder obtained from silverbellies is much lower in comparison to WAC of prawn meat (Shamasundar and Prakash, 1994). The ability of protein molecule to adsorb the added water will decrease with alteration of native structure (Hermansson, 1972). The highest value of WAC and FAC in both mix were found in $25 \%$ fish powder incorporated mix. 
Table.1 Physico-chemical, functional \& microbiological characteristics of fish mince

\begin{tabular}{|c|c|c|}
\hline S. No & Parameters & Leiognathus sp \\
\hline 1. & Moisture (\%) & $76.59 \pm 0.10$ \\
\hline 2. & Protein $(\%)$ & $16.38 \pm 0.05$ \\
\hline 3. & Fat $(\%)$ & $3.54 \pm 0.07$ \\
\hline 4. & $\operatorname{Ash}(\%)$ & $1.83 \pm 0.12$ \\
\hline 5. & TMA-N (mg \%) & Absent \\
\hline 6. & TVB-N (mg \%) & $2.03 \pm 0.11$ \\
\hline 7. & $\operatorname{FFA}(\%$ of oleic acid $)$ & $0.0061 \pm 0.02$ \\
\hline 8. & $\mathrm{PV}$ (milli equivalent $\mathrm{O}_{2} / \mathrm{kg}$ fat) & Absent \\
\hline 9. & NPN (mg/100g meat) & $207.61 \pm 0.23$ \\
\hline 10. & $\mathrm{pH}$ & $6.94 \pm 0.12$ \\
\hline 11. & Viscosity (Cp) & $11.35 \pm 0.22$ \\
\hline 12. & Solubility $(\%)$ & $90.05 \pm 0.15$ \\
\hline 13. & $\mathrm{Ca}^{2+}$ ATPase activity (( $\mu \mathrm{g}$ pi/mg protein/min) & $0.79 \pm 0.17$ \\
\hline 14. & Total plate count (TPC) (cfu/g) & $1.15 \times 10^{3}$ \\
\hline 15. & Staphylococcus aureus (cfu/g) & 7 \\
\hline 16. & E.coli $(\mathrm{cfu} / \mathrm{g})$ & 3 \\
\hline 17. & Salmonella & Nil \\
\hline 18. & Vibrio cholerae & Nil \\
\hline
\end{tabular}

Table.2 Physico- chemical and functional characteristics of fish meat powder from silver bellies (Leiognathus sp)

\begin{tabular}{|c|c|c|c|}
\hline \multirow{2}{*}{$\begin{array}{l}\text { Sl. } \\
\text { No. }\end{array}$} & \multirow[t]{2}{*}{ Parameters } & \multicolumn{2}{|c|}{ Silver bellies } \\
\hline & & $\begin{array}{l}\text { Cooked meat } \\
\text { powder }\end{array}$ & $\begin{array}{l}\text { Hydrolysed meat } \\
\text { powder }\end{array}$ \\
\hline 1. & Moisture (\%) & $6.60 \pm 0.23$ & $6.78 \pm 0.25$ \\
\hline 2. & Protein $(\%)$ & $85.52 \pm 0.21$ & $84.82 \pm 0.25$ \\
\hline 3. & Fat $(\%)$ & $2.61 \pm 0.14$ & $1.98 \pm 0.12$ \\
\hline 4. & $\operatorname{Ash}(\%)$ & $4.14 \pm 0.26$ & $5.70 \pm 0.28$ \\
\hline 5. & Calcium (mg/100g) & $344.65 \pm 1.70$ & $380.11 \pm 1.75$ \\
\hline 6. & phosphorus(mg/100g) & $390.81 \pm 2.18$ & $410.82 \pm 2.23$ \\
\hline 7. & Pepsin digestibility (\%) & $97.51 \pm 0.34$ & $96.07 \pm 0.35$ \\
\hline 8. & $\begin{array}{l}\text { Available lysine }(\mathrm{g} / 16 \mathrm{~g} \\
\text { nitrogen) }\end{array}$ & $9.94 \pm 0.16$ & $9.62 \pm 0.18$ \\
\hline 9. & WAC(g water/g dried material) & $3.12 \pm 0.02$ & $3.25 \pm 0.02$ \\
\hline 10. & FAC(g oil/g dried material) & $1.23 \pm 0.10$ & $1.10 \pm 0.10$ \\
\hline 11. & TVB-N (mg \%) & $1.35 \pm 0.05$ & $1.32 \pm 0.05$ \\
\hline 12. & FFA (\% of oleic acid) & $0.01 \pm 0.003$ & $0.009 \pm 0.002$ \\
\hline 13. & $\begin{array}{l}\text { PV (milli equivalent } \mathrm{O}_{2} / \mathrm{kg} \\
\text { fat) }\end{array}$ & $0.14 \pm 0.04$ & $0.11 \pm 0.03$ \\
\hline
\end{tabular}


Table.3 Changes in nutritional characteristics of cooked meat (Silverbellies) powder incorporated cereal mix

\begin{tabular}{|c|c|c|c|c|c|c|c|c|}
\hline Fish powder & \multicolumn{4}{|c|}{$10 \%$} & \multicolumn{4}{|c|}{$15 \%$} \\
\hline $\begin{array}{c}\text { Storage } \\
\text { period (days) }\end{array}$ & $\begin{array}{l}\text { Calcium } \\
(\mathrm{mg} / 100 \mathrm{~g})\end{array}$ & $\begin{array}{c}\text { Phosphorus } \\
\text { (mg/100g) }\end{array}$ & $\begin{array}{l}\text { Available } \\
\text { lysine } \\
\text { (g/16 g } \\
\text { nitrogen) }\end{array}$ & $\begin{array}{c}\text { Pepsin } \\
\text { digestibility } \\
(\%)\end{array}$ & $\begin{array}{l}\text { Calcium } \\
(\mathrm{mg} / 100 \mathrm{~g})\end{array}$ & $\begin{array}{c}\text { Phosphorus } \\
\text { (mg/100g) }\end{array}$ & $\begin{array}{l}\text { Available } \\
\text { lysine } \\
\text { (g/16 g } \\
\text { nitrogen) }\end{array}$ & $\begin{array}{c}\text { Pepsin } \\
\text { digestibility } \\
(\%)\end{array}$ \\
\hline 0 & $203.00 \pm 2.70$ & $305.64 \pm 3.45$ & $6.39 \pm 0.14$ & $90.17 \pm 1.25$ & $212.47 \pm 2.54$ & $\begin{array}{c}309.84 \pm \\
2.56\end{array}$ & $6.51 \pm 0.23$ & $90.69 \pm 2.35$ \\
\hline 30 & $202.40 \pm 2.51$ & $304.25 \pm 3.15$ & $5.67 \pm 0.25$ & $89.37 \pm 1.35$ & $211.83 \pm 2.64$ & $\begin{array}{c}308.37 \pm \\
2.34\end{array}$ & $5.88 \pm 0126$ & $90.27 \pm 2.68$ \\
\hline 60 & $201.81 \pm 2.16$ & $302.86 \pm 2.95$ & $4.95 \pm 0.64$ & $88.57 \pm 1.65$ & $211.21 \pm 2.36$ & $\begin{array}{c}306.90 \pm \\
2.84\end{array}$ & $5.26 \pm 0.85$ & $89.86 \pm 2.46$ \\
\hline 90 & $199.66 \pm 1.78$ & $300.01 \pm 2.56$ & $3.75 \pm 0.36$ & $87.16 \pm 1.48$ & $208.71 \pm 2.15$ & $\begin{array}{c}301.00 \pm \\
2.65\end{array}$ & $4.88 \pm 0.64$ & $88.62 \pm 2.63$ \\
\hline
\end{tabular}

\begin{tabular}{|c|c|c|c|c|c|c|c|c|}
\hline Fish powder & \multicolumn{4}{|c|}{$20 \%$} & \multicolumn{4}{|c|}{$25 \%$} \\
\hline $\begin{array}{c}\text { Parameters } \\
\begin{array}{c}\text { Storage } \\
\text { period } \\
\text { (days) }\end{array}\end{array}$ & $\begin{array}{l}\text { Calcium } \\
(\mathrm{mg} / \mathbf{1 0 0 g})\end{array}$ & $\begin{array}{c}\text { Phosphorus } \\
\text { (mg/100g) }\end{array}$ & $\begin{array}{c}\text { Available } \\
\text { lysine } \\
\text { (g/16 g } \\
\text { nitrogen) }\end{array}$ & $\begin{array}{c}\text { Pepsin } \\
\text { digestibilit } \\
\mathbf{y} \\
(\%)\end{array}$ & $\begin{array}{l}\text { Calcium } \\
(\mathrm{mg} / \mathbf{1 0 0 g})\end{array}$ & $\begin{array}{c}\text { Phosphorus } \\
\text { (mg/100g) }\end{array}$ & $\begin{array}{c}\text { Available } \\
\text { lysine (g/16 g } \\
\text { nitrogen) }\end{array}$ & $\begin{array}{c}\text { Pepsin } \\
\text { digestibility } \\
(\%)\end{array}$ \\
\hline 0 & $220.23 \pm 2.75$ & $314.94 \pm 3.52$ & $6.97 \pm 0.21$ & $91.09 \pm 1.28$ & $227.52 \pm 2.57$ & $319.23 \pm 2.58$ & $7.40 \pm 0.25$ & $91.57 \pm 2.39$ \\
\hline 30 & $219.85 \pm 2.56$ & $313.73 \pm 3.16$ & $6.53 \pm 0.28$ & $90.62 \pm 1.37$ & $226.64 \pm 2.69$ & $317.48 \pm 2.37$ & $7.02 \pm 0.15$ & $91.10 \pm 2.70$ \\
\hline 60 & $219.27 \pm 2.19$ & $312.59 \pm 2.98$ & $6.10 \pm 0.71$ & $90.16 \pm 1.68$ & $225.78 \pm 2.39$ & $315.74 \pm 2.86$ & $6.64 \pm 0.87$ & $90.62 \pm 2.47$ \\
\hline 90 & $218.51 \pm 1.80$ & $311.10 \pm 1.82$ & $4.97 \pm 0.39$ & $89.27 \pm 1.52$ & $221.90 \pm 2.79$ & $313.20 \pm 2.69$ & $5.33 \pm 0.71$ & $89.43 \pm 2.67$ \\
\hline
\end{tabular}


Table.4 Changes in nutritional characteristics of hydrolysed meat (Silverbellies) powder incorporated cereal mix

\begin{tabular}{|c|c|c|c|c|c|c|c|c|}
\hline Fish powder & \multicolumn{4}{|c|}{$10 \%$} & \multicolumn{4}{|c|}{$15 \%$} \\
\hline Parameters & \multirow{2}{*}{$\begin{array}{l}\text { Calcium } \\
(\mathrm{mg} / 100 \mathrm{~g})\end{array}$} & \multirow{2}{*}{$\begin{array}{l}\text { Phosphorus } \\
(\mathrm{mg} / 100 \mathrm{~g})\end{array}$} & \multirow{2}{*}{$\begin{array}{l}\text { Available } \\
\text { lysine } \\
\text { (g/16 g } \\
\text { nitrogen) }\end{array}$} & \multirow{2}{*}{$\begin{array}{c}\text { Pepsin } \\
\text { digestibility } \\
(\%)\end{array}$} & \multirow{2}{*}{$\begin{array}{l}\text { Calcium } \\
(\mathrm{mg} / 100 \mathrm{~g})\end{array}$} & \multirow{2}{*}{$\begin{array}{l}\text { Phosphorus } \\
(\mathrm{mg} / 100 \mathrm{~g})\end{array}$} & \multirow{2}{*}{$\begin{array}{l}\text { Available } \\
\text { lysine } \\
\text { (g/16 g } \\
\text { nitrogen) }\end{array}$} & \multirow{2}{*}{$\begin{array}{c}\text { Pepsin } \\
\text { digestibility } \\
(\%)\end{array}$} \\
\hline $\begin{array}{c}\text { Storage } \\
\text { period (days) }\end{array}$ & & & & & & & & \\
\hline 0 & $\begin{array}{c}207.36 \pm \\
2.32\end{array}$ & $\begin{array}{c}307.54 \pm \\
2.62\end{array}$ & $6.18 \pm 0.23$ & $88.19 \pm 1.32$ & $217.35 \pm 2.31$ & $312.00 \pm 2.36$ & $6.48 \pm 0.24$ & $88.98 \pm 1.35$ \\
\hline 30 & $206.68 \pm 2.65$ & $306.44 \pm 2.42$ & $5.25 \pm 0.28$ & $87.60 \pm 1.56$ & $216.77 \pm 2.65$ & $311.05 \pm 2.59$ & $6.01 \pm 0.56$ & $88.43 \pm 1.64$ \\
\hline 60 & $206.00 \pm 2.41$ & $305.36 \pm 2.31$ & $4.32 \pm 0.29$ & $87.00 \pm 1.98$ & $215.91 \pm 2.84$ & $310.12 \pm 2.41$ & $5.55 \pm 0.34$ & $87.89 \pm 1.85$ \\
\hline 90 & $201.75 \pm 2.15$ & $\begin{array}{c}303.40 \pm \\
2.19\end{array}$ & $3.92 \pm 0.27$ & $86.38 \pm 1.64$ & $212.25 \pm 2.96$ & $308.27 \pm 2.37$ & $4.64 \pm 0.14$ & $86.75 \pm 1.45$ \\
\hline
\end{tabular}

\begin{tabular}{|c|c|c|c|c|c|c|c|c|}
\hline Fish powder & \multicolumn{4}{|c|}{$20 \%$} & \multicolumn{4}{|c|}{$25 \%$} \\
\hline $\begin{array}{c}\text { Parameters } \\
\begin{array}{c}\text { Storage } \\
\text { period } \\
\text { (days) }\end{array}\end{array}$ & $\begin{array}{l}\text { Calcium } \\
(\mathrm{mg} / 100 \mathrm{~g})\end{array}$ & $\begin{array}{c}\text { Phosphorus } \\
\text { (mg/100g) }\end{array}$ & $\begin{array}{c}\text { Available } \\
\text { lysine } \\
\text { (g/16 g } \\
\text { nitrogen) }\end{array}$ & $\begin{array}{c}\text { Pepsin } \\
\text { digestibility } \\
(\%)\end{array}$ & $\begin{array}{l}\text { Calcium } \\
(\mathrm{mg} / 100 \mathrm{~g})\end{array}$ & $\begin{array}{l}\text { Phosphorus } \\
\text { (mg/100g) }\end{array}$ & $\begin{array}{l}\text { Available } \\
\text { lysine } \\
\text { (g/16 g } \\
\text { nitrogen) }\end{array}$ & $\begin{array}{c}\text { Pepsin } \\
\text { digestibility } \\
(\%)\end{array}$ \\
\hline 0 & $227.89 \pm 2.58$ & $\begin{array}{c}318.59 \pm \\
2.68\end{array}$ & $6.96 \pm 0.26$ & $89.55 \pm 1.38$ & $\begin{array}{c}236.52 \pm \\
2.34\end{array}$ & $\begin{array}{c}323.27 \pm \\
2.39\end{array}$ & $7.20 \pm 0.28$ & $90.53 \pm 1.39$ \\
\hline 30 & $226.73 \pm 2.67$ & $317.09 \pm 2.47$ & $6.32 \pm 0.31$ & $89.01 \pm 1.57$ & $235.33 \pm 2.67$ & $\begin{array}{c}321.76 \pm \\
2.64\end{array}$ & $6.61 \pm 0.59$ & $90.07 \pm 1.68$ \\
\hline 60 & $225.58 \pm 2.56$ & $\begin{array}{c}315.61 \pm \\
2.37\end{array}$ & $5.69 \pm 0.34$ & $88.47 \pm 1.99$ & $234.16 \pm 2.88$ & $\begin{array}{c}320.25 \pm \\
2.47\end{array}$ & $6.02 \pm 0.43$ & $89.61 \pm 1.89$ \\
\hline 90 & $221.70 \pm 2.27$ & $\begin{array}{c}314.20 \pm \\
2.22\end{array}$ & $4.86 \pm 0.29$ & $87.82 \pm 1.67$ & $\begin{array}{c}232.52 \pm \\
2.98\end{array}$ & $\begin{array}{c}318.37 \pm \\
2.39\end{array}$ & $5.37 \pm 0.19$ & $88.57 \pm 1.49$ \\
\hline
\end{tabular}


Table.5 Changes in functional characteristics of cooked meat (Silverbellies) powder incorporated cereal mix

\begin{tabular}{|c|c|c|c|c|c|c|c|c|}
\hline \multirow{2}{*}{$\begin{array}{c}\begin{array}{c}\text { Fish } \\
\text { powder }\end{array} \\
\text { Parameters } \\
\begin{array}{c}\text { Storage } \\
\text { Period (days) }\end{array}\end{array}$} & \multicolumn{2}{|c|}{$10 \%$} & \multicolumn{2}{|c|}{$15 \%$} & \multicolumn{2}{|c|}{$20 \%$} & \multicolumn{2}{|c|}{$25 \%$} \\
\hline & WAC & FAC & WAC & FAC & WAC & FAC & WAC & FAC \\
\hline 0 & $2.40 \pm 0.23$ & $\begin{array}{c}0.65 \pm 0.3 \\
2\end{array}$ & $2.44 \pm 0.65$ & $0.68 \pm 0.36$ & $2.48 \pm 0.47$ & $0.70 \pm 0.62$ & $2.52 \pm 0.57$ & $0.72 \pm 0.35$ \\
\hline 30 & $2.51 \pm 0.34$ & $\begin{array}{c}0.62 \pm 0.2 \\
7\end{array}$ & $2.54 \pm 0.54$ & $0.65 \pm 0.45$ & $2.59 \pm 0.51$ & $0.68 \pm 0.57$ & $2.65 \pm 0.67$ & $0.71 \pm 0.51$ \\
\hline 60 & $2.62 \pm 0.31$ & $\begin{array}{c}0.58 \pm 0.2 \\
8\end{array}$ & $2.65 \pm 0.56$ & $0.63 \pm 0.85$ & $2.70 \pm 0.37$ & $0.65 \pm 0.67$ & $2.78 \pm 0.31$ & $0.69 \pm 0.81$ \\
\hline 90 & $2.35 \pm 0.29$ & $\begin{array}{c}0.56 \pm 0.1 \\
7\end{array}$ & $2.38 \pm 0.23$ & $0.60 \pm 0.65$ & $2.57 \pm 0.64$ & $0.64 \pm 0.28$ & $2.63 \pm 0.28$ & $0.66 \pm 0.51$ \\
\hline
\end{tabular}

Table.6 Changes in functional characteristics of hydrolysed meat (Silverbellies) powder incorporated cereal mix

\begin{tabular}{c|c|c|c|c|c|c|c|c|}
\hline $\begin{array}{c}\text { Fish } \\
\text { powder }\end{array}$ & \multicolumn{2}{|c|}{$10 \%$} & \multicolumn{2}{c|}{$15 \%$} & \multicolumn{2}{c|}{$20 \%$} & & $25 \%$ \\
\hline $\begin{array}{c}\text { Parameters } \\
\begin{array}{c}\text { Storage } \\
\text { Period (days) }\end{array}\end{array}$ & WAC & FAC & WAC & FAC & WAC & FAC & WAC & FAC \\
\hline 0 & $2.56 \pm 0.34$ & $0.68 \pm 0.24$ & $2.60 \pm 0.24$ & $0.71 \pm 0.31$ & $2.64 \pm 0.35$ & $0.74 \pm 0.35$ & $2.70 \pm 0.42$ & $0.76 \pm 0.31$ \\
\hline 30 & $2.64 \pm 0.44$ & $0.66 \pm 0.37$ & $2.68 \pm 0.28$ & $0.70 \pm 0.51$ & $2.73 \pm 0.62$ & $0.72 \pm 0.25$ & $2.79 \pm 0.41$ & $0.75 \pm 0.25$ \\
\hline 60 & $2.72 \pm 0.23$ & $0.65 \pm 0.36$ & $2.77 \pm 0.62$ & $0.69 \pm 0.45$ & $2.83 \pm 0.34$ & $0.71 \pm 0.36$ & $2.88 \pm 0.32$ & $0.73 \pm 0.21$ \\
\hline 90 & $2.48 \pm 0.37$ & $\mathbf{0 . 6 3} \pm 0.53$ & $2.63 \pm 0.18$ & $0.68 \pm 0.61$ & $2.69 \pm 0.12$ & $0.69 \pm 0.81$ & $2.75 \pm 0.28$ & $0.72 \pm 0.36$ \\
\hline
\end{tabular}

WAC - Water absorption capacity (g water/g dried material)

FAC $-\mathrm{F}$ at absorption capacity (g oil / g dried material) 
Table.7 Changes in biochemical characteristics of cooked meat (Silverbellies) powder incorporated cereal mix

\begin{tabular}{|c|c|c|c|c|c|c|c|c|c|c|c|c|}
\hline $\begin{array}{l}\text { Fish meat } \\
\text { powder }\end{array}$ & \multicolumn{3}{|c|}{$10 \%$} & \multicolumn{3}{|c|}{$15 \%$} & \multicolumn{3}{|c|}{$20 \%$} & \multicolumn{3}{|c|}{$25 \%$} \\
\hline $\begin{array}{c}\text { Parameters } \\
\begin{array}{c}\text { Storage period } \\
\text { (days) }\end{array}\end{array}$ & FFA & PV & TVB-N & FFA & PV & TVB-N & FFA & PV & TVB-N & FFA & PV & $\mathbf{T V B}-\mathbf{N}$ \\
\hline 0 & $\begin{array}{c}0.04 \pm \\
0.05\end{array}$ & $\begin{array}{c}0.57 \pm \\
0.39\end{array}$ & $1.68 \pm 0.59$ & $\begin{array}{c}0.05 \pm \\
0.57\end{array}$ & $\begin{array}{c}0.71 \pm \\
0.75\end{array}$ & $\begin{array}{c}3.56 \pm \\
0.48\end{array}$ & $\begin{array}{c}0.05 \pm \\
0.06\end{array}$ & $\begin{array}{c}0.85 \pm \\
0.08\end{array}$ & $\begin{array}{c}5.19 \pm \\
0.37\end{array}$ & $\begin{array}{c}0.05 \pm \\
0.64\end{array}$ & $\begin{array}{c}0.92 \pm \\
0.37\end{array}$ & $\begin{array}{c}6.05 \pm \\
0.93\end{array}$ \\
\hline 30 & $\begin{array}{c}0.06 \pm \\
0.01\end{array}$ & $\begin{array}{c}0.78 \pm \\
0.47\end{array}$ & $3.93 \pm 0.64$ & $\begin{array}{c}0.06 \pm \\
0.62\end{array}$ & $\begin{array}{c}0.96 \pm \\
0.67\end{array}$ & $\begin{array}{c}6.41 \pm \\
0.92\end{array}$ & $\begin{array}{c}0.07 \pm \\
0.09\end{array}$ & $\begin{array}{c}1.12 \pm \\
0.57\end{array}$ & $\begin{array}{c}8.34 \pm \\
0.28\end{array}$ & $\begin{array}{c}0.07 \pm \\
0.09\end{array}$ & $\begin{array}{c}1.21 \pm \\
0.94\end{array}$ & $\begin{array}{c}9.65 \pm \\
0.54 \\
\end{array}$ \\
\hline 60 & $\begin{array}{c}0.08 \pm \\
0.02\end{array}$ & $\begin{array}{c}1.00 \pm \\
0.69\end{array}$ & $6.18 \pm 0.97$ & $\begin{array}{c}0.09 \pm \\
0.34\end{array}$ & $\begin{array}{c}1.22 \pm \\
0.38\end{array}$ & $\begin{array}{c}9.26 \pm \\
0.37\end{array}$ & $\begin{array}{c}0.09 \pm \\
0.08\end{array}$ & $\begin{array}{c}1.38 \pm \\
0.63\end{array}$ & $\begin{array}{c}11.49 \pm \\
0.91\end{array}$ & $\begin{array}{c}0.10 \pm \\
0.07\end{array}$ & $\begin{array}{c}1.51 \pm \\
0.64\end{array}$ & $\begin{array}{c}13.25 \pm \\
0.38\end{array}$ \\
\hline 90 & $\begin{array}{c}0.10 \pm \\
0.03\end{array}$ & $\begin{array}{c}1.22 \pm \\
0.92\end{array}$ & $10.78 \pm 0.54$ & $\begin{array}{c}0.11 \pm \\
0.67\end{array}$ & $1.47 \pm 0.28$ & $14.30 \pm 0.64$ & $\begin{array}{c}0.13 \pm \\
0.06\end{array}$ & $\begin{array}{c}1.65 \pm \\
0.85\end{array}$ & $\begin{array}{c}17.12 \pm \\
0.37\end{array}$ & $\begin{array}{c}0.15 \pm \\
0.09\end{array}$ & $\begin{array}{c}1.91 \pm \\
0.29\end{array}$ & $\begin{array}{c}18.97 \pm \\
0.64\end{array}$ \\
\hline
\end{tabular}

FFA-Free Fatty Acid, PV-Peroxide Value (milli equivalent of $\mathrm{O}_{2} / \mathrm{kg}$ of fat),

TVB-N-Total Volatile Base -Nitrogen ( $\mathrm{mg} \%)$.

Table.8 Changes in biochemical characteristics of hydrolysed meat (Silverbellies) powder incorporated cereal mix

\begin{tabular}{|c|c|c|c|c|c|c|c|c|c|c|c|c|}
\hline $\begin{array}{l}\text { Fish meat } \\
\text { powder }\end{array}$ & \multicolumn{3}{|c|}{$10 \%$} & \multicolumn{3}{|c|}{$15 \%$} & \multicolumn{3}{|c|}{$20 \%$} & \multicolumn{3}{|c|}{$25 \%$} \\
\hline $\begin{array}{c}\text { Parameters } \\
\begin{array}{c}\text { Storage period } \\
\text { (days) }\end{array}\end{array}$ & FFA & PV & TVB-N & FFA & PV & TVB-N & FFA & PV & TVB-N & FFA & PV & TVB-N \\
\hline 0 & $\begin{array}{c}0.04 \pm \\
0.08\end{array}$ & $\begin{array}{c}0.36 \pm \\
0.27\end{array}$ & $\begin{array}{c}1.52 \pm \\
0.34\end{array}$ & $\begin{array}{c}0.04 \pm \\
0.37\end{array}$ & $\begin{array}{c}0.43 \pm \\
0.61\end{array}$ & $\begin{array}{c}2.25 \pm \\
0.62\end{array}$ & $\begin{array}{c}0 . .04 \pm \\
0.52\end{array}$ & $\begin{array}{c}0.49 \pm \\
0.61\end{array}$ & $\begin{array}{c}3.89 \pm \\
0.68\end{array}$ & $\begin{array}{c}0.04 \pm \\
0.62\end{array}$ & $\begin{array}{c}0.58 \pm \\
0.38\end{array}$ & $\begin{array}{c}5.54 \pm \\
0.67\end{array}$ \\
\hline 30 & $\begin{array}{c}0.05 \pm \\
0.09\end{array}$ & $\begin{array}{c}0.58 \pm \\
0.39\end{array}$ & $\begin{array}{c}3.16 \pm \\
0.61\end{array}$ & $\begin{array}{c}0.05 \pm \\
0.51\end{array}$ & $\begin{array}{c}0.68 \pm \\
0.29\end{array}$ & $\begin{array}{c}4.59 \pm \\
0.38\end{array}$ & $\begin{array}{c}0.06 \pm \\
0.38\end{array}$ & $\begin{array}{c}0.75 \pm \\
0.64\end{array}$ & $\begin{array}{c}6.62 \pm \\
0.67\end{array}$ & $\begin{array}{c}0.06 \pm \\
0.39\end{array}$ & $\begin{array}{c}0.90 \pm \\
0.61\end{array}$ & $\begin{array}{c}8.56 \pm \\
0.37\end{array}$ \\
\hline 60 & $\begin{array}{c}0.07 \pm \\
0.07\end{array}$ & $\begin{array}{c}0.81 \pm \\
0.51\end{array}$ & $\begin{array}{c}4.81 \pm \\
0.92\end{array}$ & $\begin{array}{c}0.07 \pm \\
0.62\end{array}$ & $\begin{array}{c}0.94 \pm \\
0.46\end{array}$ & $\begin{array}{c}6.94 \pm \\
0.61\end{array}$ & $\begin{array}{c}0.08 \pm \\
0.67\end{array}$ & $\begin{array}{c}1.01 \pm \\
0.39\end{array}$ & $\begin{array}{c}9.36 \pm \\
0.37\end{array}$ & $\begin{array}{c}0.08 \pm \\
0.61\end{array}$ & $\begin{array}{c}1.22 \pm \\
0.92\end{array}$ & $\begin{array}{c}11.58 \pm \\
0.96\end{array}$ \\
\hline 90 & $\begin{array}{c}0.09 \pm \\
0.08\end{array}$ & $\begin{array}{c}1.05 \pm \\
0.67\end{array}$ & $\begin{array}{c}8.74 \pm \\
0.37\end{array}$ & $\begin{array}{c}0.09 \pm \\
0.63\end{array}$ & $\begin{array}{c}1.20 \pm \\
0.37\end{array}$ & $\begin{array}{c}10.86 \pm \\
0.92\end{array}$ & $\begin{array}{c}0.09 \pm 0 \\
38\end{array}$ & $\begin{array}{c}1.24 \pm \\
0.51\end{array}$ & $\begin{array}{c}14.10 \pm \\
0.68\end{array}$ & $\begin{array}{c}0.10 \pm \\
0.94\end{array}$ & $\begin{array}{c}1.63 \pm \\
0.67\end{array}$ & $\begin{array}{c}16.82 \pm \\
0.78\end{array}$ \\
\hline
\end{tabular}

FFA- Free Fatty Acid (\%Oleic acid), PV-Peroxide Value (milli equivalent of $\mathrm{O}_{2} / \mathrm{kg}$ of fat),

TVB-N-Total Volatile Base -Nitrogen (mg \%). 
Table.9 Organoleptic evaluation of drink prepared (Over all acceptability)

\begin{tabular}{|l|c|c|c|c|}
\hline & $10 \%$ & $15 \%$ & $20 \%$ & $25 \%$ \\
\hline $\begin{array}{l}\text { Cooked fish meat } \\
\text { powder + cereals }\end{array}$ & $4.63 \pm 0.42$ & $4.52 \pm 0.44$ & $4.51 \pm 0.41$ & $4.48 \pm 0.42$ \\
\hline $\begin{array}{l}\text { Hydrolysed fish meat } \\
\text { powder + cereals }\end{array}$ & $4.58 \pm 0.37$ & $4.54 \pm 0.40$ & $4.50 \pm 0.41$ & $4.47 \pm 0.42$ \\
\hline
\end{tabular}

Table.10 Changes in biochemical composition of cooked meat (Silverbellies) powder incorporated cereal mix

\begin{tabular}{|c|c|c|c|c|c|c|c|c|}
\hline Fish & \multicolumn{2}{|c|}{$10 \%$} & \multicolumn{2}{|c|}{$15 \%$} & \multicolumn{2}{|c|}{$20 \%$} & \multicolumn{2}{|c|}{$25 \%$} \\
\hline $\begin{array}{l}\text { Parameters } \\
\text { Storage } \\
\text { days }\end{array}$ & $\begin{array}{c}\text { Protein } \\
\%\end{array}$ & $\begin{array}{c}\text { Carbohydra } \\
\text { te } \%\end{array}$ & $\begin{array}{c}\text { Protein } \\
\%\end{array}$ & $\begin{array}{c}\text { Carbohydrate } \\
\%\end{array}$ & $\begin{array}{c}\text { Protein } \\
\%\end{array}$ & $\begin{array}{c}\text { Carbohydra } \\
\text { te } \%\end{array}$ & $\begin{array}{c}\text { Protein } \\
\%\end{array}$ & $\begin{array}{c}\text { Carbohydrate } \\
\%\end{array}$ \\
\hline 0 days & $18.39 \pm 0.09$ & $68.97 \pm 0.07$ & $22.03 \pm 0.22$ & $63.73 \pm 0.24$ & $24.22 \pm 0.62$ & $61.89 \pm 0.27$ & $27.91 \pm 0.61$ & $58.01 \pm 0.96$ \\
\hline 30 days & $19.14 \pm 0.64$ & $68.41 \pm 0.27$ & $23.07 \pm 0.42$ & $63.32 \pm 0.36$ & $25.11 \pm 0.42$ & $61.47 \pm 0.25$ & $27.96 \pm 0.38$ & $57.92 \pm 0.34$ \\
\hline 60 days & $19.89 \pm 0.34$ & $67.86 \pm 0.35$ & $24.12 \pm 0.34$ & $62.92 \pm 0.67$ & $26.01 \pm 0.15$ & $61.05 \pm 0.31$ & $28.02 \pm 0.17$ & $57.85 \pm 0.27$ \\
\hline 90 days & $19.63 \pm 0.28$ & $68.08 \pm 0.91$ & $23.56 \pm 0.28$ & $63.61 \pm 0.31$ & $25.79 \pm 0.42$ & $61.70 \pm 0.33$ & $28.10 \pm 0.62$ & $58.28 \pm 0.06$ \\
\hline
\end{tabular}

Table.11 Changes in biochemical composition of hydrolyzed meat (Silverbellies) powder incorporated cereal mix

\begin{tabular}{|c|c|c|c|c|c|c|c|c|}
\hline Fish & \multicolumn{2}{|c|}{$10 \%$} & \multicolumn{2}{|c|}{$15 \%$} & \multicolumn{2}{|c|}{$20 \%$} & \multicolumn{2}{|c|}{$25 \%$} \\
\hline $\begin{array}{l}\text { Parameters } \\
\text { Storage } \\
\text { days } \\
\end{array}$ & $\begin{array}{c}\text { Protein } \\
\%\end{array}$ & $\begin{array}{c}\text { Carbohydrate } \\
\%\end{array}$ & $\begin{array}{c}\text { Protein } \\
\%\end{array}$ & $\begin{array}{c}\text { Carbohydrate } \\
\%\end{array}$ & $\begin{array}{c}\text { Protein } \\
\%\end{array}$ & $\begin{array}{c}\text { Carbohydrate } \\
\%\end{array}$ & $\begin{array}{c}\text { Protein } \\
\%\end{array}$ & $\begin{array}{c}\text { Carbohydrate } \\
\%\end{array}$ \\
\hline 0 days & $16.68 \pm 0.21$ & $71.70 \pm 0.08$ & $18.62 \pm 0.87$ & $68.61 \pm 0.37$ & $21.41 \pm 0.27$ & $66.67 \pm 0.28$ & $24.95 \pm 0.34$ & $62.97 \pm 0.37$ \\
\hline 30 days & $17.32 \pm 0.23$ & $70.98 \pm 0.27$ & $19.32 \pm 0.64$ & $68.22 \pm 0.61$ & $22.12 \pm 0.21$ & $66.04 \pm 0.61$ & $25.17 \pm 0.29$ & $62.48 \pm 0.61$ \\
\hline 60 days & $17.96 \pm 0.18$ & $70.26 \pm 0.64$ & $20.03 \pm 0.54$ & $67.83 \pm 0.35$ & $22.85 \pm 0.06$ & $65.41 \pm 0.34$ & $26.00 \pm 0.45$ & $61.98 \pm 0.17$ \\
\hline 90 days & $17.68 \pm 0.34$ & $70.68 \pm 0.23$ & $19.85 \pm 0.34$ & $68.00 \pm 0.08$ & $22.38 \pm 0.09$ & $65.71 \pm 0.18$ & $25.40 \pm 0.17$ & $62.00 \pm 0.28$ \\
\hline
\end{tabular}


Table.12 Microbiological characteristics of cooked fish meat (Silverbellies) powder incorporated cereal mix

\begin{tabular}{|l|l|l|l|c|c|}
\hline & \multicolumn{5}{|c|}{ Parameter (cfu/g) } \\
\hline Storage days & \multicolumn{1}{|c|}{ TPC } & S.aureus & E.Coli & Salmonella & V. cholerae \\
\hline 0 days & $4.1 \times 10^{3}$ & $1.3 \times 10^{1}$ & Nil & Nil & Nil \\
\hline $\mathbf{3 0}$ days & $3.4 \times 10^{3}$ & $3.9 \times 10^{1}$ & Nil & Nil & Nil \\
\hline 60 days & $2.8 \times 10^{3}$ & $6.1 \times 10^{1}$ & Nil & Nil & Nil \\
\hline 90 days & $2.2 \times 10^{3}$ & $7.5 \times 10^{1}$ & Nil & Nil & Nil \\
\hline
\end{tabular}

Table.13 Microbiological characteristics of hydrolyzed fish meat (Silverbellies) powder incorporated cereal mix

\begin{tabular}{|l|l|l|l|l|l|}
\hline & \multicolumn{5}{|c|}{ Parameter (cfu/g) } \\
\hline Storage days & \multicolumn{1}{|c|}{ TPC } & S.aureus & E.Coli & Salmonella & V. cholerae \\
\hline $\mathbf{0}$ days & $4.3 \times 10^{3}$ & $1.5 \times 10^{1}$ & Nil & Nil & Nil \\
\hline $\mathbf{3 0}$ days & $3.6 \times 10^{3}$ & $3.4 \times 10^{1}$ & Nil & Nil & Nil \\
\hline $\mathbf{6 0}$ days & $2.9 \times 10^{3}$ & $5.7 \times 10^{1}$ & Nil & Nil & Nil \\
\hline $\mathbf{9 0}$ days & $2.1 \times 10^{3}$ & $6.9 \times 10^{1}$ & Nil & Nil & Nil \\
\hline
\end{tabular}


The presence of other non-protein components in the fish powder - cereal mixture may influence the WAC. FAC is purely a physical phenomenon where entrapment of added oil by macromoleculs. However it is likely that the alteration in the structure of major protein fraction may have a bearing on the FAC value (Kinsella and white head, 1989). The results for changes in biochemical characteristics of both mixtures are presented in Table 7 and 8 . The value of FFA, PV and TVB-N were found to increase with increasing amount of fish powder incorporation and Solanki et al., (1977) reported similar fact when they develop partially hydrolysed and deodourised edible fish powder. The values of TVB-N, PV, and FFA increased during storage period. FFA and TVB-N and PV content varied significantly $(\mathrm{P}<0.05)$ during storage period in cooked meat powder incorporated mixture. FFA content did not vary significantly during storage period in hydrolysed meat powder incorporated mixture whereas PV and TVB-N did vary at $1 \%$ and $5 \%$ level. The results of TVB-N, FFA \& PV in the present study are in agreement with earlier report (Chattopdhyay et al., 2004).

The value of TPC was reduced from $4.1 \times 10^{3}$ to $2.2 \times 10^{3}$ and $4.3 \times 10^{3}$ to $2.1 \times 10^{3}$ in cooked and hydrolysed meat powder incorporated cereal mixture. It is mainly due to low water activity. Salmonella, V. cholerae and E. coli were absent throughout the storage period. The published literature on the sensory qualities of concentrated fish protein products are concerned with the presence of odour and flavour components and the influence residual lipids on odour reversion during storage (Sikorski, 1981).

In the present study, organoleptic quality revealed that (Table 9) the tastes of the liquid drinks found to be with no fish odour immediately. However there is a significant difference $(\mathrm{P}<0.01)$ observed in their overall acceptability (Table 10-13)

From the results it is concluded that cereal mixture with $10 \%$ hydrolyzed meat added was acceptable organoleptically throughout the study period. Organoleptic quality revealed that the tastes of the liquid drinks found to be with no fish odour immediately Further study is required to recommend this mixture as health drink.

\section{References}

Anon., 2001. Indian marine product rises again. Seafood Export Journal., 32(6): 7-12.

AOAC, 1995. Official method of Analysis, $16^{\text {th }}$ edn. Association of official analytical chemists, Washington, D.C.

APHA, 1976. Compendium of methods for the microbiological examination of foods, Speck M.L., (ed), APHA, New York.

Beatty, S.A., and Gibbons., 1937. The measurement of spoilage in fish. J. Biol Board. Can., 3(1): 77-91.

Chattopadhyay, A.K., Madhusudana Roa, B., and Gupta, S., 2004. A simple process for the utilization of small bony fish as edible fish powder. Fish Technol., 41(2): 117-120.

CMFRI, 2006. Central Marine Fisheries Research Institute, Annual report 200506, ICAR, India Fiske, C.H., and Subbarow, Y., 1925. The colorimetric determination of phosphorus. $J$. Biol.Chem, 66: 375-380.

Feldhusen, F (2000). The role of seafood in bacterial food borne diseases. J. Microb. Infection. 2, $1651-1660$.

Hermansson, A.M., 1972. Functional properties for foods. Labensmith - Wiss Technol. 5:24

Jeyakumari, A., and Rathnakumar, K., 2006. Effect of incorporation of frozen stored 
minced fish on the quality of gelatinized product from rice and maize flour. Fish Technol., 43(2): $146-153$.

Jeyakumari, A., Rathnakumar, K. and Velauytham, P. (2006). Storage behaviour of washed mince from pink perch (Nemipterus japonium) at $-30^{\circ} \mathrm{C}$. Fish. Technol. 43(2): pp.138-145.

Kinsella, J.E., and Whitehead, D.M., 1989.

Protein in whey: chemical, physical and functional properties. Adv. Food Nutr. Res., 33:343-438

Lin, M.Y.J., Humert, E.S. and Sosulski, F.W., 1974. Certain functional properties of Sunflower meal product. J. Food Sci., 39: $368-370$

Noguchi, S and Matsumoto, J.J. (1970)

Studies on the control of denaturation of the fish muscle proteins during the frozen storage. 1. Preventive effect of Na-glutamase. Bull. Jap. Soc. Sci. Fish., 36: pp. $1078-1087$.

Olley, T., and Lovern, J.A., 1960. Phospholipid hydrolysis of cod flesh stored at various temperature, J. Sci. Food Agric., 11 (11): 644 - 652.

Revankar, G.D., Keshava., Naidu., A.K. and Baligo, B.L., 1981. Fish mince preparation and Composition. Indian Food Pack., 35(4): 20-24

Sadasivam, S., and Manickam, A., 1992. Determination of total carbohydrate by anthrone method. In: Biochemical methods for Agricultural Sciences, Wiley Eastern Limited, PP.8-9.
Sahoo, P.R., 2007. By-catch reduction devices in fisheries. Aqua Inter, 2: 2326.

Schlegel, G.H., 1992. General microbiology, $7^{\text {th }}$ ed. Cambridge university press, V.K. 2329.

Setty, R.T.M., Sudhakara, N.S., Nagaraj, A.S., and Shetty, H.P.C., 1977. Development of partially hydrolysed and deodourised fish powder. Fish Technol., 14(2): 103-108.

Sikorski, Z.E., 1981. CRC crt. Rev. Fd. Sci. Nutr. 14, 3.

Snedecor, G.W. and W.G. Cochran (1962) Factorial experiments, In: Statistical methods. Oxford and IBH publishing Co., Calcutta, pp. 339 - 380.

Solanki., K.K., Devadasan, K., and Venkataraman, R., 1977. Edible fish powder from dhoma (Sciaenids Spp), Fish Technol., 14(1): 39-43.

Solsulski, F.W., 1962. The centrifuge method for determining water absorption in hard red spring wheats. Cereal Chem., 39: 344-350.

Srinivasan, R., 1966. On the production and nutritive value of fish meal from silver belly (Leiognathus Spp) landings at Rameswaram. Fish Technol., 3(1):5258.

Venugopalan, N., and James, A.M., 1969. Utilisation of trash fish, studies on preparation of fish soup mix. Fish Technol., 6(2): 148 - 152.

\section{How to cite this article:}

Rathnakumar, K. and Pancharaja, N. 2018. Development of Health Mix from Silverbellies and their Nutritional Characteristics. Int.J.Curr.Microbiol.App.Sci. 7(03): 3090-3101. doi: https://doi.org/10.20546/ijcmas.2018.703.359 